INT-PUB 02-27

\title{
Strangeness Nucleation in Neutron Star Matter
}

\author{
Travis Norsen \\ Dept. of Physics, University of Washington, Seattle, WA 98195
}

(October 29, 2018)

\begin{abstract}
We study the transition from npe-type nuclear matter (consisting of neutrons, protons, and electrons) to matter containing strangeness, using a Walecka-type model predicting a first-order kaon-condensate phase transition. We examine the free energy of droplets of K-matter as the density, temperature, and neutrino fraction are varied. Langer nucleation rate theory is then used to approximate the rate at which critical droplets of the new phase are produced by thermal fluctuations, thus giving an estimate of the time required for the new (mixed) phase to appear at various densities and various times in the cooling history of the proto-neutron star. We also discuss the famous difficulty of "simultaneous weak interactions" which we connect to the literature on non-topological solitons. Finally, we discuss the implications of our results to several phenomenological issues involving neutron star phase transitions.
\end{abstract}

\section{INTRODUCTION}

A 'hot' topic recently in nuclear physics has been the attempt to understand the production of strangeness in the expanding plasma thought to be created in relativistic collisions of heavy ions. There are several aspects to this complex and difficult problem, including: how (and, indeed, whether) a 3-flavor deconfined quark-gluon plasma is produced by the initial collision; how this strange matter equilibrates, expands, and cools; and how it eventually re-hadronizes, hopefully giving rise to a unique observable signal. The goal of these studies is to better understand the phase structure of QCD in the low-density, high-temperature region of the phase diagram.

A different but complementary problem involves the study of nuclear matter at highdensity and low temperature, such as that existing in neutron star interiors. Here, matter consisting of neutrons, protons, and electrons (npe matter) is crushed during the gravitational collapse of the parent star. (Actually, what we call npe matter in this paper may also include muons and neutrinos.) As the star collapses weak interactions convert electrons and protons into neutrons, with electron neutrinos produced copiously as a by-product. Additionally, neutrinos of all flavors are created through brehmstrahlung-type collisions among 
the warm nucleons. As the neutrinos diffuse outward and radiate away, the proto-neutron star cools from an initial temperature of several tens of $\mathrm{MeVs}$, and eventually settles into the familiar ground state of a neutron star with central density several times nuclear matter density.

It is now understood that at sufficiently high density, a phase transition will occur in which the neutrons and protons of npe matter are replaced by deconfined quarks (including strange quarks). [1 [3] A related mechanism for the appearance of strangeness in neutron star matter is kaon condensation, which may become possible at densities somewhat lower than the density required for the deconfining transition described above. The possibility of kaon condensation was first pointed out by Kaplan and Nelson, who were motivated by the chiral Lagrangian prediction of an attractive interaction between $K^{-}$and nuclear matter. [1]

Intuitively, one can understand the phenomenon of kaon condensation in the following way. As the density of npe matter is increased, and assuming that all particle species are in equilibrium with respect to the weak interactions, the electron chemical potential increases. Simultaneously, the effective mass of an in-medium $K^{-}$will decrease, due to the attrative interaction mentioned above. Therefore, at some critical density, the electron chemical potential (i.e., the energy of the electrons at the top of the Fermi sea) will become greater than the effective mass of the $K^{-}$. It then becomes energetically favorable for the high-energy electrons to decay according to

$$
e^{-} \rightarrow \nu_{e}+K^{-}
$$

at which point a condensate of $K^{-}$will form.

This picture is somewhat over-simplified, as the production of kaons does not proceed exclusively by the decay of energetic electrons, but may also occur via fully-hadronic weak couplings, e.g., $n \rightarrow p+K^{-}$. Also, the notion of a uniform condensate of kaons is probably wrong. As pointed out by Glendenning [5], the presence of two separate conserved charges in neutron star matter (namely, baryon number and electric charge) gives rise to the possibility of a mixed phase of $\mathrm{N}$-matter and K-matter existing over a wide range of densities and pressures in the star. (By "N-matter" we mean matter not containing kaons, but without the restriction of charge neutrality implied by "npe matter".) This mixed phase will have the form of a Coulomb lattice - the now standard "pasta" structure consisting of droplets of the new phase immersed in a background of N-matter (at low densities), with rods and slabs replacing droplets as the density is increased, and finally with the role of the two phases interchanging in the transition toward homogenous K-matter as the density is increased still further. [13

In this paper, we will assume a model which predicts just this kind of first-order phase transition to kaon-condensed matter. For the description of nuclear matter, we use a Walecka-type model in which the interactions of strongly-interacting particles are mediated by $\sigma, \omega$, and $\rho$ mesons, treated in the mean-field approximation. Kaons are included in the model on the same footing as the nucleons, as described in the next section.

Our goal is, however, not to study the structure of the ground state predicted by this model (and its subsequent effect on the global neutron star properties such as the massradius relation), but rather to study the nucleation of kaons, that is, the process by which the initial proto-neutron star matter with zero strangeness acquires strangeness through the 
spontaneous appearance of K-matter droplets. We will begin this study by using the theory of homogenous nucleation due to Langer. [6] Here one assumes that at a given temperature, there are constant fluctuations producing small short-lived droplets of the new phase. Below a certain critical size, the surface energy cost of these droplets wins out over the volume term and the droplets shrink away. For some critical size, however, the free energy gained from the production of a large volume of the new (energetically favored) phase is just large enough to cancel the cost of surface energy, and the droplet will spontaneously grow. In the case studied here, because the two phases have non-zero electric charge densities, there is also a Coulomb term in the free energy, which becomes large for large droplets. Hence, a super-critical droplet will not grow forever, but will reach a stable size at which the energy gain between the volume and surface energies just balances the energy cost of the Coulomb energy.

Langer showed that the expected time needed for this kind of phase transition to proceed depended on the probability of a critical droplet being produced by thermal fluctuations, and also on the growth rate of such a critical droplet along the unstable direction in configuration space. Assuming a thermal distribution of these fluctuations, the nucleation rate per unit volume can be written

$$
\Gamma \sim I_{0} e^{-\Delta F\left(R_{\text {crit }}\right) / T}
$$

where $\Delta F\left(R_{\text {crit }}\right)$ is the excess free energy of a critical droplet, $T$ is the temperature, and $I_{0}$ (the "prefactor") is a microscopic fluctuation rate related to the growth rate of a supercritical droplet, the thermal conductivity of the medium, and other properties. [6 \& The prefactor is often approximated by dimensional analysis as simply $I_{0} \sim T^{4}$. We will discuss this approximation later. Our goal is to apply this nucleation rate theory to the case of cooling proto-neutron star matter in order to better understand exactly when, where, and how the transition from npe-type matter to the kaon-condensed phase occurs.

Our work generally follows previous work on the nucleation of quark matter droplets in neutron star matter, which has been studied extensively. 9 12 The case of kaon condensation is physically unique, however, since it is the only realistic proposed example of a direct first order transition from npe-type neutron star matter to matter containing strangeness. In the case of the deconfinement transition mentioned above, for example, and even at densities, temperatures, and pressures for which 3-flavor quark matter is energetically favored, the transition is indirect in the following sense: an intermediate stage of 2-flavor quark matter will be produced first, with strange quarks then slowly appearing during a smooth crossover or second-order transition to the 3-flavor ground state. This latter transition is, of course, slow due to the weakness of the weak interactions. In this scenario, however, the original nucleation events which take one from npe matter to deconfined quark matter, need not involve the weak interactions at all.

The case of kaon condensation is radically different, since here there is no intermediate zero-strangeness state which might allow for fast nucleation followed by a slow but smooth growth of the strangeness-containing fields. Instead, the thermal fluctuations responsible for nucleation events must directly involve the weak-interaction processes which produce kaons. The difficulties posed by the widely varying timescales involved in these two sorts of processes (thermal fluctuations and weak interactions) will form the theme of our discussion.

The outline of the rest of the paper is as follows. In Section II we present the details of 
the nuclear mean field theory used for the subsequent discussion. In Section III we use this model to extract information about the free energy of K-matter droplets of different sizes, as the background baryon number density and temperature are varied. This allows us to use Eq. (2) to estimate the nucleation rate for the kaon-condensate phase transition. In Section $\square$ we discuss in more detail the underlying mechanism for the fluctuations which produce the nucleation, and thereby analyze the trustworthiness of the estimates. Here we also make contact with the literature on Q-balls and argue that the problem of direct strangeness nucleation in neutron star matter may be profitably considered as an instance of Q-ball nucleation. Finally, in Section $\nabla$ we summarize the findings, discuss the relevance of our results to phenomenological issues in neutron star physics, and indicate some proposals for future investigation.

\section{MEAN-FIELD THEORY DESCRIPTION OF KAON CONDENSATION}

In this section we briefly review the model proposed by Glendenning and Schaffner [13 which predicts a first-order transition from nuclear matter to the kaon-condensed phase. The model begins with a relativistic Walecka-type Lagrangian describing the neutron and proton fields, as well as the $\sigma, \omega$, and $\rho$ mesons which mediate their interactions:

$$
\begin{aligned}
\mathcal{L}_{N}= & \bar{\Psi}_{N}\left(i \gamma^{\mu} \partial_{\mu}-m_{N}^{*}-g_{\omega N} \gamma^{\mu} V_{\mu}-g_{r N} \gamma^{\mu} \vec{\tau}_{N} \cdot \vec{R}_{\mu}\right) \Psi_{N} \\
& +\frac{1}{2} \partial_{\mu} \sigma \partial^{\mu} \sigma-\frac{1}{2} m_{\sigma}^{2} \sigma^{2}-U(\sigma)-\frac{1}{4} V_{\mu \nu} V^{\mu \nu} \\
& +\frac{1}{2} m_{\omega}^{2} V_{\mu} V^{\mu}-\frac{1}{4} \vec{R}_{\mu \nu} \cdot \overrightarrow{R^{\mu \nu}}+\frac{1}{2} m_{r}^{2} \vec{R}_{\mu} \cdot \vec{R}^{\mu}
\end{aligned}
$$

where $m_{N}^{*}=m_{N}-g_{\sigma N} \sigma$ is the nucleon effective mass, which is reduced compared to the free nucleon mass due to the scalar field $\sigma$. The vector fields corresponding to the omega and rho mesons are given by $V_{\mu \nu}=\partial_{\mu} V_{\nu}-\partial_{\nu} V_{\mu}$, and $\vec{R}_{\mu \nu}=\partial_{\mu} \vec{R}_{\nu}-\partial_{\nu} \vec{R}_{\mu}$ respectively. $\Psi_{N}$ is the nucleon field operator with $\vec{\tau}_{N}$ the nucleon isospin operator.

In addition to the usual kinetic, mass, and interaction terms for the nucleon and meson fields, the model also includes cubic and quartic self-interactions of the $\sigma$ field:

$$
U(\sigma)=\frac{1}{3} b m_{N}\left(g_{\sigma N} \sigma\right)^{3}+\frac{1}{4} c\left(g_{\sigma N} \sigma\right)^{4}
$$

where $b$ and $c$ are dimensionless coupling constants. These coupling constants (as well as the three nucleon-meson couplings: $g_{\sigma N}, g_{\omega N}$, and $\left.g_{r N}\right)$ are chosen to reproduce the empirical properties of nuclear matter at saturation density. [14, 15]

Kaons are included in the model in the same fashion as the nucleons, by coupling to the $\sigma, \omega$ and $\rho$ meson fields. There exist in the literature several meson-exchange Lagrangians which attempt to describe kaon-nucleon interactions. A detailed discussion of these models and their relation to the Chiral Lagrangian description proposed by Kaplan and Nelson [4] can be found in papers by Pons et al. [16] and Prakash et al. [17,.18]. In the present paper we employ the Lagrangian proposed by Glendenning and Schaffner. [13] The kaon Lagrangian is given by

$$
\mathcal{L}_{K}=\left(\mathcal{D}_{\mu} K\right)^{\dagger}\left(\mathcal{D}^{\mu} K\right)-m_{K}^{* 2} K^{\dagger} K
$$


where $K$ denotes the isospin doublet kaon field. The covariant derivative $\mathcal{D}_{\mu}=\partial_{\mu}+i g_{\omega K} V_{\mu}+$ $i g_{r K} \vec{\tau}_{K} \cdot \vec{R}_{\mu}$ couples the kaon field to the vector mesons and the kaon effective mass term $m_{K}^{*}=m_{K}-g_{\sigma K} \sigma$ describes its coupling to the scalar meson. $\vec{\tau}_{K}$ is the kaon isospin operator.

The vector coupling constants are determined by isospin and quark counting rules [13] and are given by $g_{\omega K}=g_{\omega N} / 3$ and $g_{r K}=g_{r N}$. The scalar coupling is fixed by fitting to an empirically determined kaon optical potential in nuclear matter. The real part of this quantity has been determined from properties of kaonic atoms to lie in the somewhat wide range $80 \mathrm{MeV} \lesssim U_{K}\left(n_{o}\right) \lesssim 180 \mathrm{MeV}$ [19],20]. Here, we choose $U_{K}\left(n_{o}\right)=120 \mathrm{MeV}$ to fix $g_{\sigma K}$. Lower values of the kaon optical potential reduce the strength of the first-order transition and, eventually, produce instead a second-order phase transition, while higher values make the first-order transition stronger. While the issues discussed in this paper rely on the existence of a first-order transition, the qualitative conclusions are generally independent of the specific value of the coupling. So long as the transition is first-order, the main effect of changing $U_{K}\left(n_{o}\right)$ will be to change the critical density for the onset of the mixed phase, without severely affecting our discussion of the nucleation properties near this critical density.

The model as presented so far is a complicated, strongly interacting field theory which cannot be solved in any reasonable way. It is therefore standard to make a mean-field approximation, in which the meson field operators are replaced by their expectation values. Because of rotational invariance only the time-component of the vector fields $V_{\mu}$ and $\vec{R}_{\mu}$ can have a non-zero expectation value. Likewise, only the isospin 3-component of the isovector field $\vec{R}_{\mu}$ can be non-zero. The equations of motion for the meson (mean-) fields can be simply derived from the above Lagrangians and are given by:

$$
\begin{aligned}
m_{\sigma}^{2} \sigma & =-\frac{d U}{d \sigma}+g_{\sigma B}\left(n_{n}^{(s)}+n_{p}^{(s)}\right)+g_{\sigma k} m_{K}^{*} f_{K}^{2} \theta^{2} \\
m_{\omega}^{2} \omega & =g_{\omega N}\left(n_{n}+n_{p}\right)-g_{\omega K} f_{K}^{2} \theta^{2}\left(\mu_{K}+g_{\omega K} \omega+g_{r K} r\right) \\
m_{r}^{2} r & =g_{r N}\left(n_{p}-n_{n}\right)-g_{r K} f_{K}^{2} \theta^{2}\left(\mu_{K}+g_{\omega K} \omega+g_{r K} r\right)
\end{aligned}
$$

where the meson fields $\sigma, \omega, r$ now represent the appropriate mean values. Here $n_{n}$ and $n_{p}$ represent the neutron and proton number densities, respectively, while $n_{n}^{(s)}$ and $n_{p}^{(s)}$ are the corresponding scalar densities. We have substituted $K=\left(0, K^{-}\right)$and $K^{-}=\frac{1}{\sqrt{2}} f_{K} \theta e^{-i \mu_{K} t}$, where $f_{K}$ is the kaon decay constant and $\theta$ is a dimensionless kaon field strength parameter. We have neglected to write down the additional small contributions to the kaon-coupling terms due to finite temperature effects, though these are included in our code. (See Ref. [16] for a more explicit presentation of the details of this model for finite temperature.) We will be working in the bulk approximation (in which the meson fields do not vary with position) and so have set to zero the gradient terms which would otherwise appear in the equations of motion.

The equation of motion for the kaon field (in terms of $\theta$ ) is

$$
0=\left(\left(m_{K}^{*}\right)^{2}-\left(\mu_{K}^{*}\right)^{2}\right) \theta
$$

which indicates that the kaon effective mass $m_{K}^{*}=m_{K}-g_{\sigma K} \sigma$ and the effective chemical potential $\mu_{K}^{*}=\mu_{K}+X$ must be equal in order for the kaon field to take on a non-zero value. Here $X=g_{\omega K} \omega+g_{r K} r$ is the vector field contribution to the $K^{-}$energy. We also 
include a Langrangian describing non-interacting spin- $\frac{1}{2}$ particles to account for the presence of electrons, muons, and neutrinos.

The thermodynamic potential per unit volume for the nucleon sector is

$$
\begin{aligned}
\frac{\Omega_{N}}{V}= & \frac{1}{2} m_{\sigma}^{2} \sigma^{2}+U(\sigma)-\frac{1}{2} m_{\omega}^{2} \omega^{2}-\frac{1}{2} m_{r}^{2} r^{2} \\
& -2 T \sum_{i=n, p} \int \frac{\mathrm{d}^{3} k}{(2 \pi)^{3}} \ln \left(1+e^{\left(E(k)-\nu_{i}\right) / T}\right)
\end{aligned}
$$

where the nucleon energy $E(k)=\sqrt{k^{2}+m_{N}^{*}}$. The chemical potentials are given by $\mu_{p}=$ $\nu_{p}+g_{\omega N} \omega+\frac{1}{2} g_{r N} r$ and $\mu_{n}=\nu_{n}+g_{\omega N} \omega-\frac{1}{2} g_{r N} r$.

The other thermodynamic quantities can be calculated from $\Omega_{N}$ in the standard way. The nucleonic contribution to the pressure, for example, is $P_{N}=-\Omega_{N} / V$, while the number densities and entropy densities are given by

$$
\begin{aligned}
n_{n, p} & =-\frac{\partial \Omega_{N} / V}{\partial \mu_{n, p}} \\
& =2 \int \frac{\mathrm{d}^{3} k}{(2 \pi)^{3}} f_{F}\left(E(k)-\nu_{n, p}\right) \\
s_{n, p} & =-\frac{\partial \Omega_{N} / V}{\partial T}
\end{aligned}
$$

where $f_{F}(\epsilon)=\left(e^{-\epsilon / T}+1\right)^{-1}$ is the Fermi-Dirac distribution function. The neutron and proton scalar densities which enter in the equation of motion for the $\sigma$ field are given by

$$
n_{n, p}^{(s)}=2 \int \frac{d^{3} k}{(2 \pi)^{3}} \frac{m_{N}^{*}}{E(k)} f_{F}\left(E(k)-\nu_{n, p}\right)
$$

The energy density is determined through the usual relation $T s_{N}=\epsilon_{N}+P_{N}-\sum_{i} \mu_{i} n_{i}$ to be

$$
\begin{aligned}
\epsilon_{N}= & \frac{1}{2} m_{\sigma}^{2} \sigma^{2}+U(\sigma)-\frac{1}{2} m_{\omega}^{2} \omega^{2}-\frac{1}{2} m_{r}^{2} r^{2} \\
& +2 \sum_{i=n, p} \int \frac{\mathrm{d}^{3} k}{(2 \pi)^{3}} E(k) f_{F}\left(E(k)-\nu_{i}\right) \\
& +\sum_{i=n, p} n_{i}\left(\mu_{i}-\nu_{i}\right) .
\end{aligned}
$$

The thermodynamic potential for the lepton species present (electrons, muons, and neutrinos) is given by

$$
\frac{\Omega_{L}}{V}=-\sum_{l} T g_{l} \int \frac{\mathrm{d}^{3} k}{(2 \pi)^{3}}\left[\ln \left(1+e^{-\left(E_{l}(k)-\mu_{l}\right) / T}\right)+\ln \left(1+e^{-\left(E_{l}(k)+\mu_{l}\right) / T}\right)\right]
$$

where $\mu_{l}$ denotes the chemical potential for lepton species $l$, and $g_{l}$ are the spin-degeneracies: $g=2$ for electrons and muons, $g=1$ for neutrinos. $\beta$-equilibrium requires the following constraints on the chemical potentials: 


$$
\mu_{K}=\mu_{e}-\mu_{\nu_{e}}=\mu_{n}-\mu_{p}=\mu_{\mu}-\mu_{\nu_{\mu}}
$$

The lepton contributions to the pressure, energy density, and entropy are determined from Eq.(16) in the usual way.

Finally, the thermodynamic potential for the kaons is given by

$$
\begin{aligned}
\frac{\Omega_{K}}{V}= & \frac{1}{2} f_{K}^{2} \theta^{2}\left[\left(m_{K}^{*}\right)^{2}-\left(\mu_{K}+X\right)^{2}\right] \\
& +T \int \frac{d^{3} p}{(2 \pi)^{3}} \ln \left(1-e^{-\left(\omega^{-}(p)-\mu_{K}\right) / T}\right)
\end{aligned}
$$

where $\omega^{-}(p)=\sqrt{p^{2}+\left(m_{K}^{*}\right)^{2}}-X$ is the in-medium energy of a $K^{-}$with momentum $p$. Again, the kaon contribution to the other thermodynamic quantities can be found by differentiation. For a detailed derivation and presentation of the thermodynamics of this model, see Ref. [16]. For discussions of similar studies involving mixed-phases in mean-field-theories in several different physical contexts see, e.g., 252 28].

Our goal here is to study the rate at which K-matter nucleates in a background of npe-matter at various temperatures and densities. As discussed previously, this involves calculating the free energy of droplets of various sizes, in particular, the free energy of the critical droplet configuration. The first step toward this end is to produce a description of a droplet of K-matter of arbitrary radius. This involves solving the meson field equations of motion subject to various constraints.

Generally, one solves simultaneously two versions of the meson field equations above: one with $\theta=0$ describing the $\mathrm{N}$-matter component of the mixed phase, the other with $\theta \neq 0$ (with the specific value of $\theta$ determined self-consistently through Eq. (90) describing the K-matter. One requires chemical equilibrium between the two phases, i.e., that the relevant chemical potentials in the two phases match. Additionally, the requirement of overall electric charge neutrality means that the electric charge densities in the two phases must be of opposite sign. One then uses the respective charge densities to calculate $\alpha=$ $-q^{(N)} /\left(q^{(K)}-q^{(N)}\right)$, the volume fraction of the K-matter phase. (Here $q^{(N)}$ and $q^{(K)}$ are the charge densities of the K-matter and N-matter, respectively.) Because we are concerned with droplets of small radius, it is also crucial to include correct mechanical equilibrium between the two phases. In Ref. [23] this requirement was shown to affect rather dramatically the bulk properties of the two phases. (See also [24].) For a spherical droplet of K-matter in a background of $\mathrm{N}$-matter, this constraint reads:

$$
P_{K}-P_{N}=2 \sigma / R
$$

where $\sigma$ is the surface tension between the two phases, and $R$ is the radius of the droplet.

Finally, it should be noted that we work at constant baryon number density. That is, in constructing a sequence of droplets of varying radii, we require that the overall baryon density $n_{B}=\alpha n_{B}^{(K)}+(1-\alpha) n_{B}^{(N)}$ be held fixed at some specified value. Here $n_{B}^{(N, K)}$ are the baryon number densities of the N- and K-phases.

Once the equations of motion are solved self-consistently subject to these constraints, it is possible to calculate the free energy of a given droplet. The total bulk energy density of a given droplet configuration is defined analogously to the overall baryon number density: 


$$
\epsilon_{\text {bulk }}=\alpha \epsilon^{(K)}+(1-\alpha) \epsilon^{(N)}
$$

where the energy density in each phase includes the contributions from the nucleons, leptons, and kaons, as appropriate. The total bulk energy is then found by multiplying this energy density by the total volume of a Wigner-Seitz cell (defined as the spherical region including one droplet and containing zero total electric charge), $V_{W S}$. This energy is then supplemented by the surface and Coulomb contributions:

$$
E(R)=\epsilon_{b u l k} V_{W S}(R)+4 \pi R^{2} \sigma+E_{C o u l}
$$

where $E_{\text {Coul }}$ is found by integrating the electric field energy density $\epsilon_{C o u l}(r)=\frac{1}{2}|E(r)|^{2}$ throughout the Wigner-Seitz cell. (Here $|E(r)|$ is the magnitude of the electric field determined by Gauss' Law.) The total entropy of a droplet configuration is found analogously, by volume-fraction-weighted averaging of the individual phase entropy densities, then multiplying by the volume of the Wigner-Seitz cell. Thus, the total free energy of a droplet can be calculated as:

$$
F(R)=E(R)-T S(R) .
$$

Only the R-dependence has been indicated explicitly, but, of course, the energy and entropy both depend strongly on the fixed baryon number density, the temperature, neutrino fraction, etc.

We are interested, however, not merely in the free energy of a given configuration, but, rather, the change in free energy required to produce various droplets. Hence, we also solve for pure, charge neutral npe matter at a given baryon density and calculate its free energy density, $f_{n p e}=\epsilon-T s$. We are then led to define

$$
\Delta F(R)=F(R)-f_{n p e} V_{W S} .
$$

which represents the free energy cost of a transition from electrically neutral npe matter (the initial state of an evolving proto-neutron star) to a single droplet of kaon-condensed matter of radius $R$. Fig. 1 1 shows $\Delta F(R)$ as a function of $R$ near the critical density for the transition, for two different values of the surface tension, $\sigma=20 \mathrm{MeV} / \mathrm{fm}^{2}$ and $\sigma=30 \mathrm{MeV} / \mathrm{fm}^{2}$.

We have shown the free energy curves for two values of $\sigma$ in order to illustrate the role this quantity plays. As expected, smaller values of $\sigma$ reduce both the size and free energy cost of a critical droplet. In what follows, we will simply pick the value $\sigma=30 \mathrm{MeV} / \mathrm{fm}^{2}$, a value suggested by Glendenning's study of the boundary between the two phases [21], as well as by our own earlier work on this model [23].

In the following section we will use this type of curve to acquire information about the free energy cost of a critical droplet under various conditions of temperature, density, and neutrino fraction.

\section{DROPLET FREE ENERGY AND NUCLEATION RATES}

As mentioned in the introduction, one can understand the production of kaons in the neutron star matter as being due to the weak decay of an electron (or, equivalently, the 

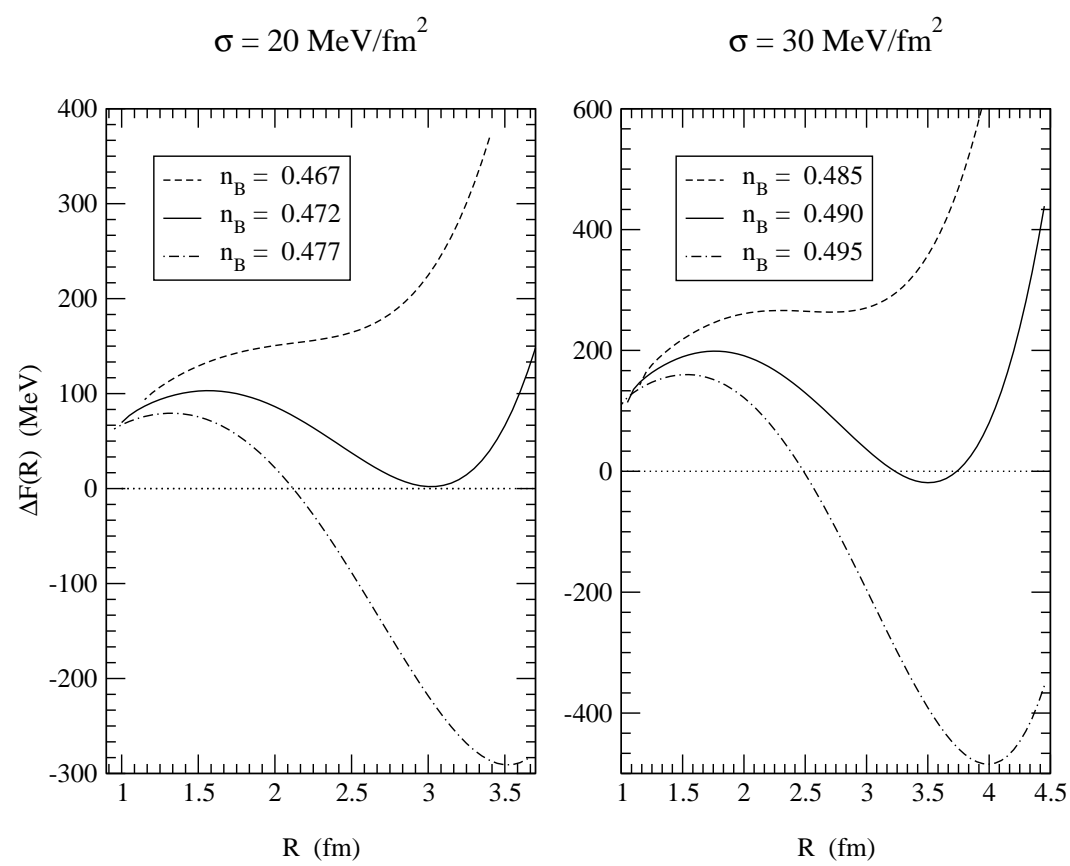

FIG. 1. Free Energy cost of kaon droplets (as a function of the radius) for two different values of the surface tension, $\sigma$. Each plot is shown near the critical density for the transition (which is slightly different for the two values of $\sigma$ ), with additional curves drawn just above and just below the critical density, showing the development of an energetically favored droplet structure as the density is increased past the critical density.

change of a neutron into a proton plus a kaon). By definition, above the critical density this transition is energetically favorable, i.e., exothermic. So we may schematically write

$$
e^{-} \rightleftharpoons \nu_{e}+K^{-}+\text {Heat. }
$$

The excess heat generated by the production of kaons will eventually be radiated away in the form of photons and neutrinos as the neutron star cools, but that is not our interest here. Rather, our goal is to understand how the free energy of droplet configurations depends on the temperature. Qualitatively, one can guess the correct answer by applying LeChatelier's principle to the equilibrium indicated in Eq.(24): raising the temperature will tend to push the equilibrium back toward the left. That is, raising the temperature should lower the energetic favorability of kaon droplets.

This prediction is borne out by our calculations, as illustrated in Fig. 2. As the temperature is increased from zero, the free energy of kaon droplet configurations increases relative to that of neutral npe matter at the same density and temperature. This increase (which is as large as several hundred $\mathrm{MeV}$ for typical droplets) means that, at a density where the mixed phase would be the ground state at $T=0$, the mixed phase is no longer favored at higher temperature. In other words, turning up the temperature increases (slightly) the critical density for the transition.

However, because the kaon phase droplets must be produced by thermal fluctuations, we expect the thermal nucleation rate of the kaon phase to be much larger at higher temperatures. In order to estimate the speed with which droplets of the kaon phase are produced, we use the Langer nucleation rate theory discussed in the introduction. The nucleation rate 


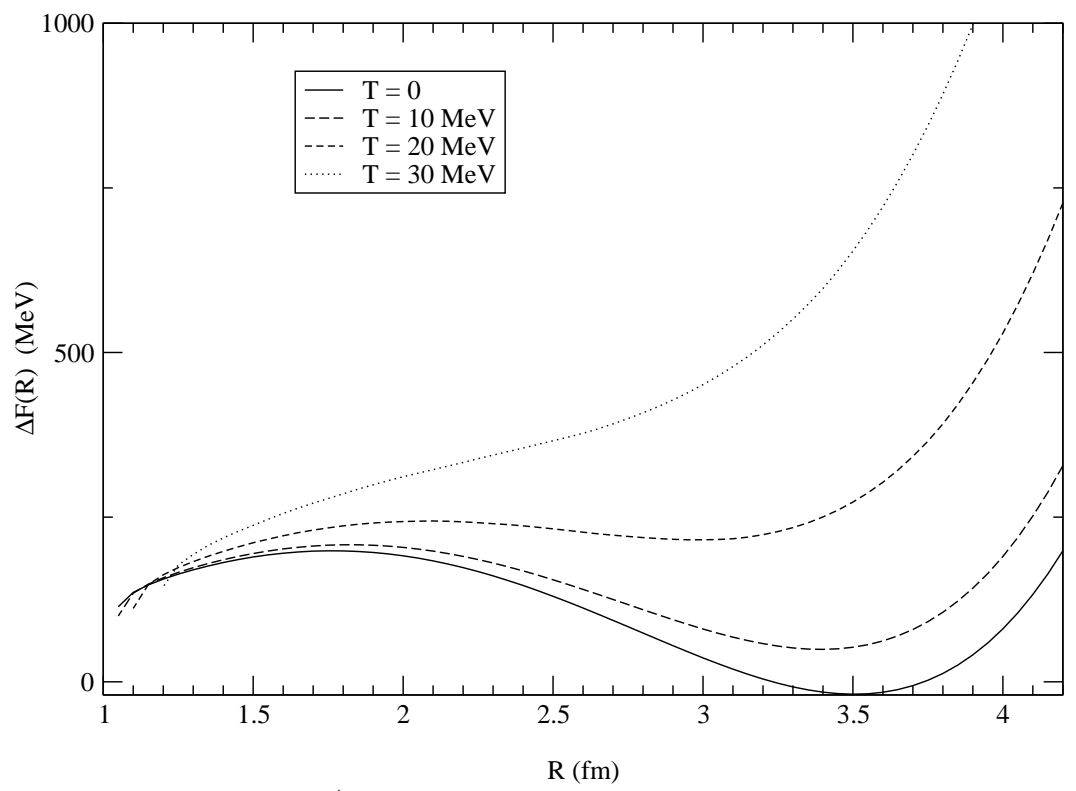

FIG. 2. Free energy of droplets (relative to neutral npe matter with the same baryon number density) as a function of radius at temperatures varying between zero and $30 \mathrm{MeV}$. As expected, increasing the temperature decreases the energetic favorability of the kaon phase.

per unit volume is estimated as:

$$
\Gamma \sim T^{4} e^{-\Delta F\left(R_{\text {crit }}\right) / T}
$$

where $\mathrm{T}$ is the temperature and $R_{\text {crit }}$ is the critical droplet radius (at a given temperature and baryon density). The free energy cost of this critical droplet configuration (i.e., the height of the barrier which must be crossed to produce a stable droplet of the new phase) can be easily read off of graphs like the ones already shown. One may then plug directly into Eq. (25) to give the nucleation rate per unit volume. In order to convert this rate into an intuitively meaningful quantity, we calculate the expected time for a single nucleation event in a single typical Wigner-Seitz cell of volume $V_{W S}=10^{3} \mathrm{fm}^{3}$. This time is given by

$$
\tau=\frac{1}{\Gamma V_{W S}}=\frac{e^{\Delta F\left(R_{c r i t}\right) / T}}{V_{W S} T^{4}}
$$

In Fig. [3 we show this nucleation time as a function of density for several different temperatures. (Note the log scale!) As expected, the nucleation time is a very strong function of the temperature. At a temperature of $0.1 \mathrm{MeV}$, the expected nucleation time is many, many times longer than the age of the universe across the entire density range of the mixed phase. At $T=1.0 \mathrm{MeV}$ the nucleation time is prohibitively long at the lower end of the mixed phase density regime, but is less than one second at densities above about $n_{B} \sim 0.55 \mathrm{fm}^{-3}$, which is something like $\frac{1}{2} n_{o}$ above the critical density. At higher temperatures, the nucleation proceeds almost immediately across the entire density range.

Naively, this leads to the conclusion that the neutron star settles into its ground state without any delay as it cools down from an initial temperature of several tens of $\mathrm{MeV}$. In the initial, hot conditions, thermal fluctuations are sufficiently fast and sufficiently numerous to seed K-matter droplets wherever those droplets are energetically favored. The sizes and 
$\mathrm{T}=0.1 \mathrm{MeV}$
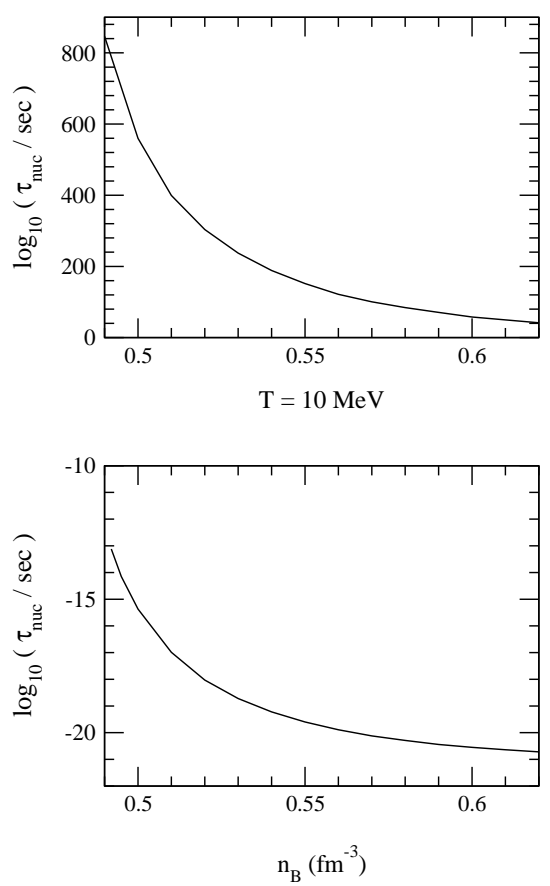

$\mathrm{T}=1.0 \mathrm{MeV}$
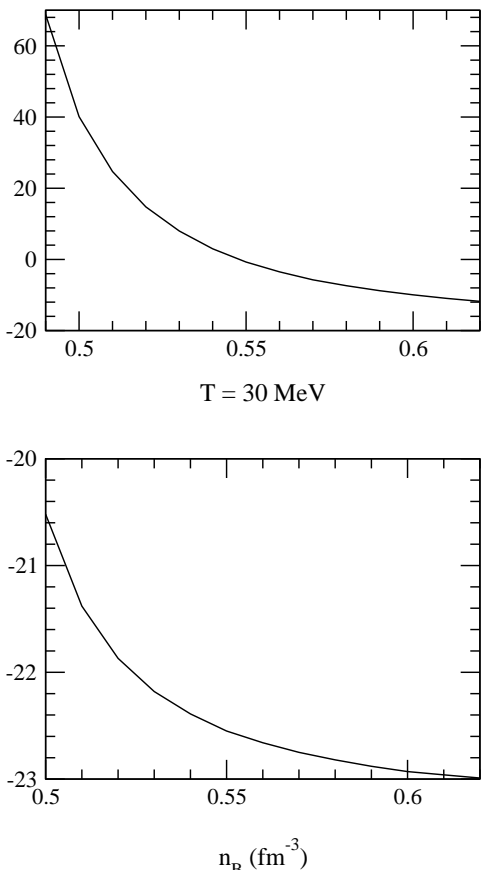

FIG. 3. Expected time for the nucleation of a single K-matter droplet in a typical Wigner-Seitz volume. Shown is the $\log _{10}$ of the nucleation time in seconds as a function of baryon number density, at several different temperatures.

distances between adjacent kaon structures may undergo tiny changes as the matter cools, and perhaps the outer edge of the mixed phase extends outward somewhat as the critical density decreases, but generally the neutron star includes the full mixed phase from birth.

This picture is complicated by at least two factors which have not been discussed explicitly until now. The first of these is the presence, in the early stages of proto-neutron star cooling, of a significant neutrino fraction. The second question is whether or not we should believe the rate estimates just given, since the nucleation rate prefactor (estimated above as $T^{4}$ ) describes the rate of microscopic fluctuations, which, in the present case, consist of weak interaction processes. These processes are, after all, weak, so one might doubt that the naive estimate based simply on dimensional analysis is appropriate. This issue will be discussed in a subsequent section; for now, we will turn to the question of the effects of neutrinos on the nucleation of K-matter. (For a related discussion see Ref. [22])

Returning to Eq.(24) and again applying LeChetalier's principle, we guess that the presence of a non-zero density of electron neutrinos will (like high temperature) suppress the appearance of K-matter. This guess turns out to be correct, but for slightly complicated reasons. Turning on a non-zero electron neutrino fraction

$$
Y_{\nu_{e}}=\frac{n_{\nu_{e}}}{n_{B}}
$$

forces the chemical potentials for electrons, kaons, and the baryons to adjust according to the constraint of constant $n_{B}$ and $\beta$-equilibrium, Eq. (17). Surprisingly, doing this actually lowers the free energy cost of kaon droplets, as indicated in Fig. 4 . 


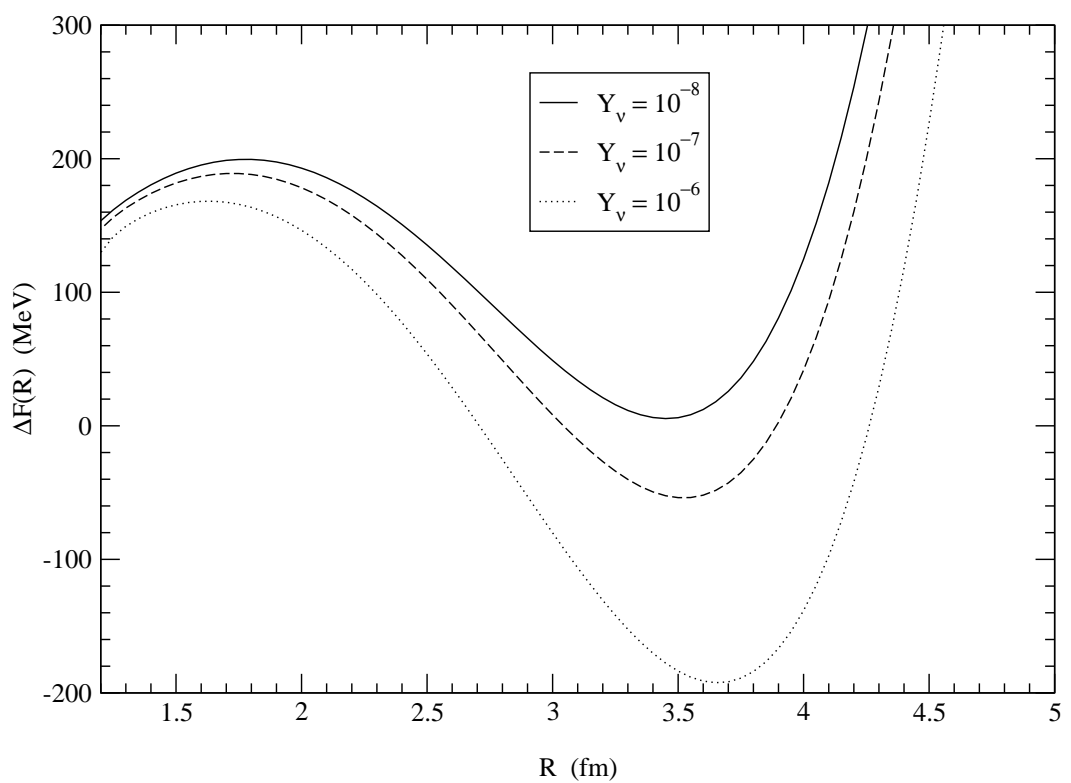

FIG. 4. Free energy of droplets (relative to neutral npe matter with the same baryon number density) as a function of radius for various small neutrino densities.

The reason for this can be understood as follows. The main effect of turning on a chemical potential for neutrinos, is to increase as well the chemical potential for electrons since the constraint of fixed baryon number density doesn't allow $\mu_{n}$ and $\mu_{p}$ much freedom to adjust. But increasing the number density of electrons decreases the (positive) electric charge density of the N-matter outside a kaon droplet. Hence, more of this matter is needed to cancel the negative charge of the droplet itself, and the volume fraction of K-matter, $\alpha$, decreases. But the overall free energy density depends on $\alpha$ through Eq.(20). Finally, since the energy density of K-matter is somewhat larger than that for electrically neutral npe matter (due not only to the presence of kaons, but also to the higher local baryon density) while that of the surrounding N-matter is somewhat lower than neutral npe matter (due to the correspondingly lower baryon number density here), the overall energy density of the mixed phase is actually decreased by the decrease in $\alpha$ coming from the non-zero neutrino density.

However, because of the requirement of overall electric charge neutrality and the fact that the K-matter is always negatively charged, the N-matter component outside of the droplet must have a positive electric charge density. As we have just seen, the presence of even a small non-zero chemical potential for electron neutrinos also increases the density of electrons, and thereby reduces the positive charge in this region. As expected, a larger neutrino fraction will decrease the electric charge density here even more, and eventually a point is reached at which the charge density ceases to be positive. Then it is no longer possible to define a Wigner-Seitz cell, i.e., no longer possible to satisfy the requirement of electric charge neutrality.

Actually, this point is reached for very moderate values of the neutrino fraction, especially at low densities (near the critical density for the transition) where, by definition, the electric charge density in the N-matter phase is positive, but small. We find that the pressure equilibrium condition Eq.(19) also has a comparable effect on the electric charge densities via the electron chemical potential. (This issue was discussed in Ref. [23].) In particular, 


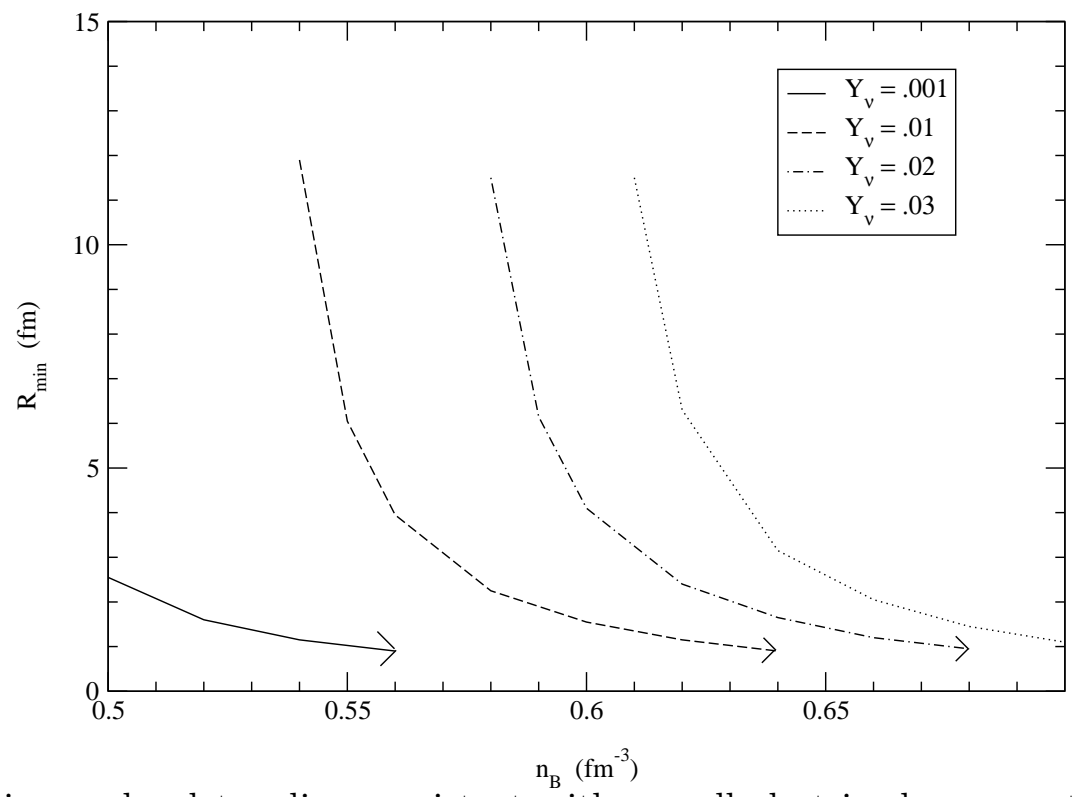

FIG. 5. Minimum droplet radius consistent with overall electric charge neutrality, as a function of baryon number density, for several different values of the neutrino fraction $Y_{\nu}$. Here the temperature is fixed at $T=0$.

larger structures at a given baryon density have lower electron chemical potentials; hence it is possible to elude the effect of non-zero $Y_{\nu}$ by producing larger K-matter droplets. Thus, at a given $Y_{\nu}$ and a given baryon density, there will be a minimum size $R_{\text {min }}$ consistent with charge neutrality, and such structures are therefore not allowed. Electron Debye screening will also play a role in forbidding structures with large radii, so the region of $n_{B}-Y_{\nu}$ parameter space in which no mixed phase can exist is even somewhat larger than suggested here. [23]

We show in Fig. 5 this minimum radius as a function of baryon density, for several different values of the electron neutrino fraction, $Y_{\nu}$. At a given $Y_{\nu}$, the minimum radius allowed by global charge neutrality begins to diverge as one comes down in density. The density at which this quantity diverges (or, if we were to consider also the effects of Debye screening, the density at which this quantity exceeds 5-10 fm) acts as an effective critical density for the onset of the mixed phase. Hence, early in the evolution of the proto-neutron star (PNS), when the neutrino fraction is (at least) a few percent, no mixed phase will be produced at densities less than $0.60-0.65 \mathrm{fm}^{-3}$. This is to be compared to the critical density at zero temperature and zero neutrino fraction, $0.49 \mathrm{fm}^{-3}$. Actually, the electron neutrino fraction in the first seconds of PNS evolution may be closer to ten percent, in which case the effective critical density below which no stable kaon mixed phases exists will be pushed upward to around $0.80 \mathrm{fm}^{-3}$, several factors of $n_{0}$ above the nominal $\left(T=0, Y_{\nu}=0\right)$ critical density. The upshot is that, in the neutrino-rich conditions of the early PNS evolution, the critical density for the onset of the kaon mixed phase is increased significantly compared to the nominal critical density.

This obviously modifies the naive inference from Fig. 3 that the mixed phase will be produced quickly and easily during the early, hot part of the PNS evolution. Because the high temperatures occur at a time of high neutrino density, there is a wide range of densities over which no mixed phase can be formed at these early times. Hence, due to 
the suppression of K-matter by neutrinos, a PNS with central density not too far above the nominal critical density for the onset of the mixed phase may fail to produce any Kmatter during its cooling, until the temperature is so low that nucleation can not occur in reasonable times. This suggests a scenario in which the neutron star could exist indefinitely in a metastable state. However, further analysis is required to support this hypothesis - in particular, we must address the question of the reliability of the nucleation rates in Fig. 3 given the problem of simultaneous weak interactions. [2]

\section{FLUCTUATIONS AND THE PROBLEM OF SIMULTANEOUS WEAK INTERACTIONS}

As a first guess, one might suppose that the nucleation of K-matter droplets is driven by thermal fluctuations in the local number density of baryons. At a sufficiently high baryon number density, the phase transition to the kaon condensed phase becomes second order, and the kaon field may grow smoothly without having to overcome an energy barrier. Hence, one might suppose that the first-order transition could occur by this same mechanism operating locally: a thermal fluctuation produces a baryon overdensity in a local region of radius $1-2 \mathrm{fm}$. The kaon field then spontaneously "fills in" this region, producing a stable droplet of K-matter which could then grow to the stable size. In this picture, the nucleation rate would be governed by the frequency of sufficiently over-dense and sufficiently large baryon number fluctuations.

However, there is an immediate and fatal problem with this basic picture. Sufficient fluctuations in baryon density are no-doubt plentiful. A typical $1-2 \mathrm{fm}$ region contains of order ten baryons, so assuming simple $\sqrt{N}$ fluctuations, a factor of two overdensity is only three standard deviations away from the mean. But the lifetime of such a fluctuation is limited to strong interaction timescales. Indeed, in nuclear matter at these densities, the speed of sound is a sizable fraction of the speed of light, $c$. Hence, the lifetime of a baryon number density fluctuation is of order $\tau \sim R / c \sim 10^{-23} \mathrm{sec}$. As is well-known from studies of a second-order kaon condensate phase transition, however, the time-scales needed for the development of an appreciable value of the kaon field are $10-15$ orders of magnitude longer than this. [29] Similar conclusions are found for the appearance of strange quark matter from an initial state of 2-flavor quark matter in neutron stars. [30.31] That is, not surprisingly, the time-scale for the development of the kaon field is typical of the weak interactions.

The production of K-matter droplets, therefore, cannot be driven by density fluctuations in the background of baryons. The microscopic kaon-production rate is simply too slow to keep up with the strong interaction timescales that govern such fluctuations, so long before any appreciable value for the kaon field in the fluctuation has developed, the fluctuation evaporates.

We conclude that the nucleation rate will be goverened by thermal fluctuations producing fluctuations in the local kaon number density itself. Moreover, so long as the timescale for the spontaneous growth of a critical droplet is small compared to weak interaction timescales, this rate can depend only on the thermal number density of kaons,

$$
n_{K}^{(\text {thermal })}=\int \frac{d^{3} p}{(2 \pi)^{3}} f_{B}\left(\omega^{-}(p)-\mu_{K}\right)
$$




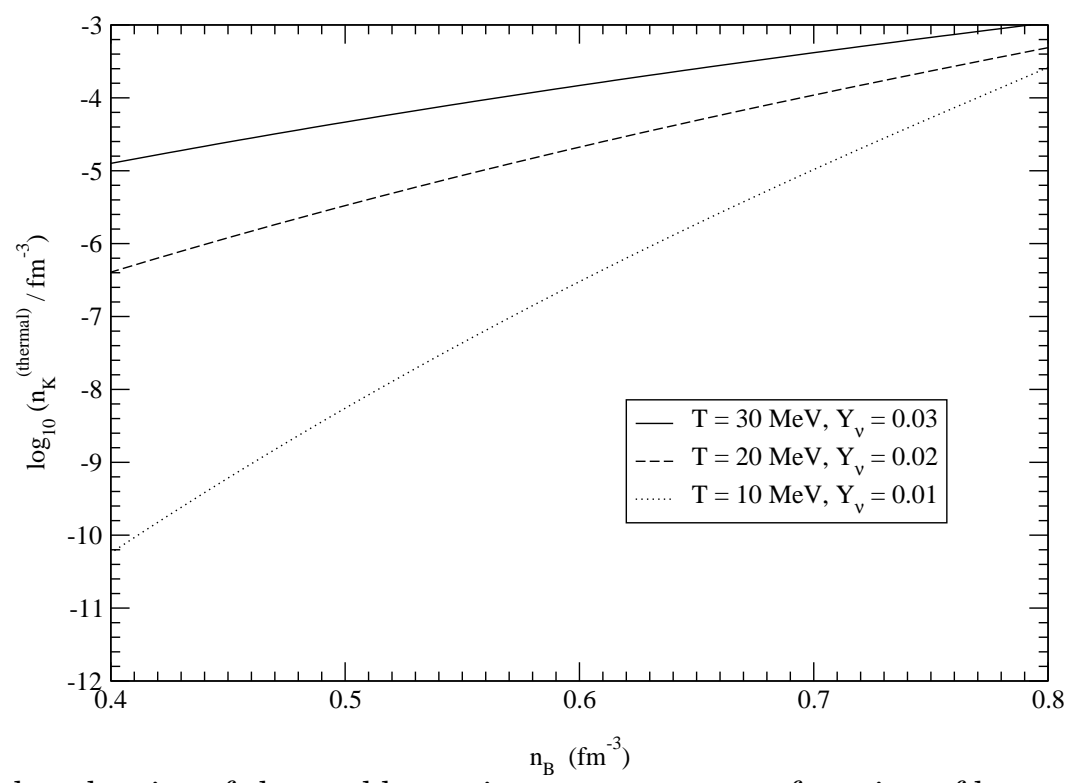

FIG. 6. Number density of thermal kaons in npe-matter as a function of baryon number density. The three curves correspond (very) approximately to three times during the cooling history of the proto-neutron star. As the star cools, the density of thermal kaons drops substantially and is effectively zero in the context of the probability of seed-production considered below.

where $f_{B}$ is the Bose-Einstein occupation probability and $\omega^{-}(p)$ was defined in Sect. [I]. We have shown in Fig.6 this thermal kaon number density for several values of the temperature and neutrino fraction in npe-type neutron star matter as a function of density.

It is then simple to estimate the probability of a sufficient "seed" of kaons appearing. This calculation is reminiscent of the standard undergraduate statistical mechanics problem of calculating the probability that all of the atoms in a box of gas are found to be in a certain small region of the box. Here, we must calculate the probability that a certain number $N$ of the kaons in a kaon gas of density $n_{K}^{(t h e r m a l)}$ spontaneously appear in some small region of space with volume $V$.

For definiteness, consider a large box of volume $V_{0}$ and total kaon number $N_{0}$, with $N_{0} / V_{0}=n_{K}^{(\text {thermal })}$. If we treat the kaons as classical particles, then the probability of $N$ kaons being found in a small sub-region $V$ of $V_{0}$ is given by the binomial theorem:

$$
P(N)=\frac{N_{0} !}{N !\left(N_{0}-N\right) !}\left(\frac{V}{V_{0}}\right)^{N}\left(1-\frac{V}{V_{0}}\right)^{\left(N_{0}-N\right)}
$$

Assuming $V \ll V_{0}$ and $N \ll N_{0}$, we may approximate $N_{0}$ ! $\sim\left(N_{0}-N\right) ! N_{0}^{N}$ and neglect $N$ in the exponent $\left(N_{0}-N\right)$. This gives

$$
P(N) \sim \frac{1}{N !}\left(\frac{N_{0} V}{V_{0}}\right)^{N}\left(1-\frac{V}{V_{0}}\right)^{N_{0}}
$$

But $N_{0} V / V_{0}=\langle N\rangle$, where $\langle N\rangle=V n_{K}^{(\text {thermal })}$ is the average number of thermal kaons in the volume $V$. Using $\langle N\rangle / V=N_{0} / V_{0}$ we have finally

$$
P(N) \sim \frac{<N>^{N}}{N !}\left(1-\frac{<N>}{N_{0}}\right)^{N_{0}}
$$




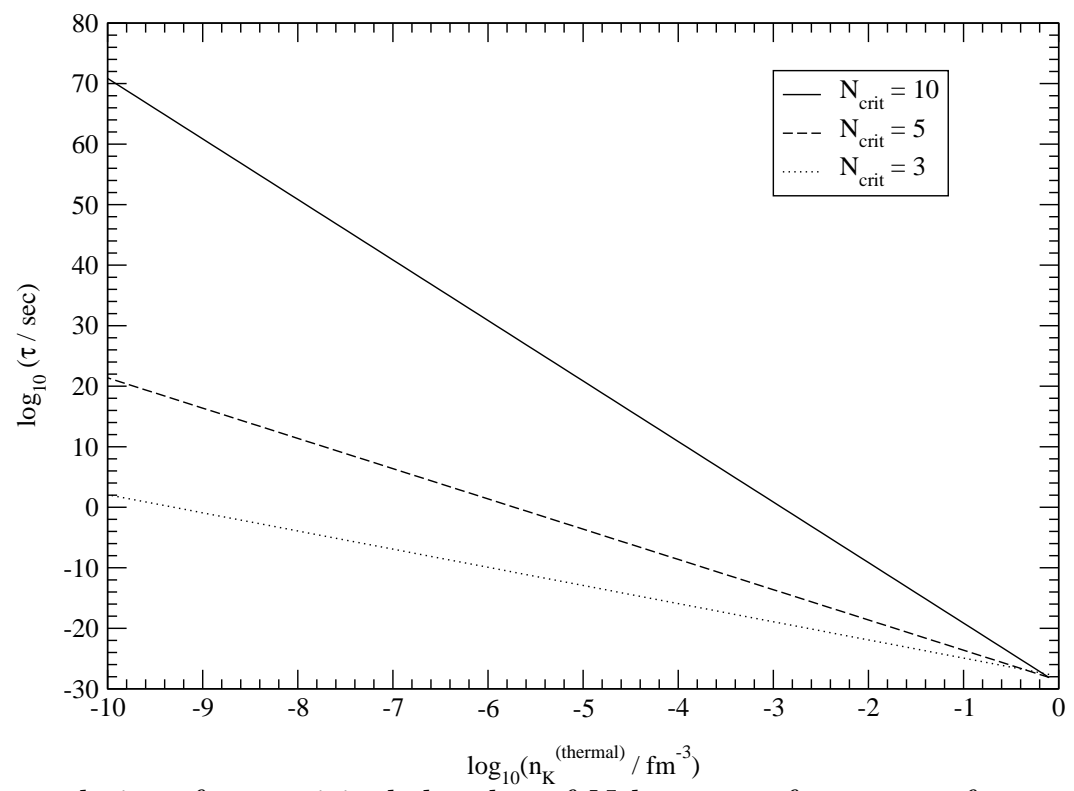

FIG. 7. Expected time for a critical droplet of $\mathrm{N}$ kaons to form out of a spontaneous density fluctuation of kaons with average density $n_{K}^{(\text {thermal })}$.

$$
\sim \frac{<N>^{N}}{N !} e^{-<N>}
$$

(In practice, $n_{K}^{(t h e r m a l)} \ll 1 \mathrm{fm}^{-3}$ so the exponential in the last line above can be ignored.)

The number of kaons in a critical droplet varies somewhat with density. Near the critical density (at zero temperature and zero neutrino fraction) the radius of a critical droplet is a few $f m$, for a critical kaon number of order 100 . At higher densities, the critical radius drops to only $1-2 \mathrm{fm}$, and the corresponding critical kaon number drops to only a few. In Fig. 7 we plot the time needed to form such a critical seed (for various $N$ ) as a function of thermal kaon number density. To estimate the seed-production time from the probability considered above, we multiply each probability by the maximum possible number of discrete seeding locations within a typical Wigner-Seitz cell. This is of order $V_{W S} n_{B} \sim 1000 \mathrm{fm}^{3} \cdot .5 / \mathrm{fm}^{3} \sim 500$. We then assume that the arrangement of thermal kaons is "reshuffled" on the fastest possible time-scale, say $\tau \sim 10^{-23} \mathrm{sec}$. (Note that this assumption is extremely generous. The actual reshuffling time must be at least several orders of magnitude greater than this, but here a generous upper limit on the seed-production rate will suffice.) Then the typical time needed for a seed of $N$ kaons to be produced by this method is given by

$$
\tau(N) \sim \frac{10^{-23} \text { sec }}{500 \cdot P(N)}
$$

with $\mathrm{P}(\mathrm{N})$ given as a function of $n_{K}^{(\text {thermal })}$ above. Actually, we should replace $P(N)$ here with $\sum_{n \geq N} P(n)$ but in practice each term in the sum is negligible compared to the one previous, so the difference will not affect the result.

We see that the number $N_{\text {critical }}$ is indeed critical to the determination of the rate. If ten (or more) kaons are needed to produce a critical droplet, the probability of a sufficient number all showing up in the same place at the same time is extremely small, and one must 
wait prohibitively long for a seed to ever be produced, especially at lower temperatures where $n_{K}^{(\text {thermal })}$ is extremely small. At higher densities where $N_{\text {crit }}$ is lower, of order 3-5, the probability is much higher the time needed for a seed to be produced by random reshuffling may be of order seconds or less for reasonable values of the temperature.

Comparing Fig. 6 and Fig. 77, we see that the time needed for a seeding event during the initial hot proto-neutron star configuration $\left(T=30 \mathrm{MeV}, Y_{\nu}=0.03\right)$ is on the order of a few seconds or less if $N_{\text {crit }} \leq 5-10$. However, as we see from Fig. 5, electric charge neutrality forbids droplets smaller than $\sim 2 \mathrm{fm}$ for densities below $\sim 0.65 \mathrm{fm}^{-3}$. This is well above the $\left(T=0, Y_{\nu}=0\right)$ critical density, and it is likely that such a high density is not reached in the proto-neutron star core, especially during the early evolution when the star is still hot and relatively rarefied.

After a few tens of seconds, the PNS temperature drops to $T=10 \mathrm{MeV}$ with the neutrino fraction also dropping to, say, $Y_{\nu}=0.01$. Looking again at Fig. 6, we see that the number density of thermal kaons has dropped dramatically due to the lower temperature. The electric charge neutrality constraint is relaxed somewhat, with droplets of radius $1-2 \mathrm{fm}$ now being allowed at densities below about $n_{B} \sim 0.58 \mathrm{fm}^{-3}$. There is a possibility, considering Fig. [7, that seeding may occur with reasonable speed for densities above this value, where $N_{\text {crit }}$ is of order $3-5$. But again, there is a fairly rigid effective critical density below which nucleation cannot occur. As the star continues to cool, the number density of thermal kaons drops dramatically, and the seeding time becomes prohibitively long at all but the very highest densities, $5-7$ times nuclear matter density, reasonably thought to exist in the neutron star core.

The crude estimates made here for seed production in a homogenous but fluctuating background of thermal kaons are similar to the estimates in [32,33] of the growth process for sub-critical Q-balls in a scalar field with a conserved charge. These authors assume a thermal distribution of sub-critical Q-balls with relatively large N, and then consider the rate at which additional charge is accreted to (and released by) the Q-ball via a random-walk process in order to estimate the rate at which critical droplets are produced. In our case, the number of kaons in a critical droplet is not much greater than unity, so we use the Poisson statistics of Eq. (31) rather than a Gaussian distribution with $\sqrt{N}$ fluctuations.

Actually, there is an exact analogy between the physics of kaon nucleation and the problem of Q-ball nucleation, i.e., the decay of metastable field configurations in the presence of a conserved charge. 34 37 The effective theory for the kaon field in our model will be precisely the theory of a complex scalar with an effective potential supporting nontopological solitons, i.e., stable droplets of K-matter. In the limit of infinitely slow weak interactions, this effective theory will contain a global $U(1)$ flavor symmetry corresponding to the conservation of strangeness. In this limit, the seeding of K-matter droplets will occur via a process formally identical to that described in Ref. [37], namely, a small uniform initial charge density (in our case, the background of thermal kaons) producing the required "bounce" configuration by flowing toward a seeding center. As discussed in [37], the action associated with this configuration will be significantly greater than the corresponding case for a real scalar which carries no conserved charge. The typical nucleation rates in the case of a charged scalar will thus be exponentially slower, due to the need for a global redistribution of charge.

The two estimates of nucleation rates in the present paper can be thought of as limits 
in the cases of infinitely slow and infinitely fast weak interactions. In reality, of course, the weak interactions proceed at some intermediate speed. In the effective theory of the kaon field described above, inclusion of strangeness-changing weak reactions will correspond to the addition of a small symmetry-breaking term in the effective potential, e.g.,

$$
U_{\text {eff }}(|K|) \rightarrow U_{\text {eff }}(|K|)+\lambda K
$$

where $\lambda$ is a strongly temperature-dependent constant that characterizes the rate at which kaons can be produced/absorbed by background scattering processes involving nucleons and leptons.

The actual rate of K-matter nucleation will depend on both types of symmetry breaking, that is (1) the initial background thermal kaon density and (2) the dynamical production of new kaons, though it is not obvious which mechanism will dominate the nucleation for physically relevant temperatures. In order to treat this problem reliably, one must construct realistic bounce configurations in the kaon effective theory as the initial background density and dynamical breaking parameter $\lambda$ are varied with temperature, impose imaginarytime periodicity corresponding to the (inverse) temperature, and calculate the action of the bounce. Qualitatively, one expects the resulting nucleation times to be intermediate between the fast-weak-interactions and no-weak-interactions limits in the current paper. At very least, one should expect that the slowness of the weak interactions should modify the nucleation rate prefactor $I_{0}$ to a more realistic dimensional analysis estimate

$$
I_{0} \rightarrow G_{F}^{2} T^{8} \sim \frac{T^{8}}{M_{W}^{4}}
$$

where $M_{W}$ is the mass of the weak gauge bosons. At a temperature of $10 \mathrm{MeV}$, this estimate would reduce the expected nucleation time by a factor of approximately $10^{-16}$ from the times shown in Fig. 3. As mentioned above, the issue of nearly-conserved strangeness will also affect the exponential factor in the rate equation, thus suppressing the rate even further and perhaps bringing the results closer to the estimates in Fig. 7. Only the reliable calculation outlined above (and currently underway by the present author) will answer this issue with any certainty, however.

\section{DISCUSSION AND CONCLUSIONS}

We have, then, an intriguing possible scenario in which the PNS manages to settle into its ground state without forming the kaon-condensate mixed phase which is the true ground state of the system. At high initial temperatures, the nucleation of K-matter droplets is suppressed by the presence of neutrinos, even though at these high temperatures the seeding of droplets is in principle fast. Over a wide range of densities kaon droplets are produced copiously by thermal fluctuations, but they are not yet stable due to the increase in effective critical density. As the star cools, the restriction coming from the presence of neutrinos is relaxed, but the intrinsic fluctuation rate drops.

For an initial PNS core density that is not too far above the nominal $(T=0)$ critical density for the formation of a K-matter mixed phase, therefore, it is likely that the star will cool not into the true ground state, but, rather, into a meta-stable state consisting of 
electrically neutral npe matter. This scenario may be relevant to understanding various phenomenological issues.

For example, the apparent existence of anomalously heavy neutron stars with masses $M \sim 2 M_{\text {sun }}$ 38 40 might be explained by the anomalously stiff equation of state of npetype matter relative to matter with a kaon condensate. Generally, if the various possible phase transitions thought to occur in neutron star matter can be avoided by the impossibility (or, equivalently, extreme slowness) of nucleation of the new phase, a relatively stiff equation of state may sometimes be maintained over a more extended range of density than would be expected naively. Hence, the existence of such heavy stars may not be sufficient evidence to rule out the existence of kaon-condensation at $3-5 n_{0}$, especially if these stars were born with smaller masses and only subsequently (that is, once cold) acquired larger masses via accretion from companion stars.

Additionally, metastability of the sort introduced above may potentially be useful in understanding the properties of GRB's or other poorly-understood explosive events. A relatively light PNS, as we have argued, may cool and deleptonize without the K-matter mixed phase forming, even when the star's core density exceeds the critical density for the transition. As is evident from Fig. 6, however, the thermal kaon density increases monotonically and steeply with density, so that, even at the very low temperatures $T \ll 1$ $\mathrm{MeV}$ eventually attained in the neutron star, there is some density at which seeding may become possible in a reasonable time. At very least, with increasing density, one eventually encounters the second-order point at which the kaon field may be produced smoothly with no need for seeding. Hence, if an initially metastable neutron star begins to accrete matter from a companion binary (or, additionally, if an initially rotating metastable neutron star gets spun down via accretion) the central density may increase sufficiently for K-matter to begin to appear.

Once the kaon matter appears, however, there will be a feedback effect, due to the softening of the equation of state. The production of a small quantity of K-matter in the core would allow the star to contract slightly, thus increasing the density in the core, and increasing the size of the region in which kaonic matter can appear. Further kaon production leads to further collapse, and vice versa. Thus if mass accretion and/or spin down results in the critical density for the onset of a second-order kaon-condensate transition being reached in the neutron star core (or, equivalently, if one reaches the low-temperature effective critical density at which a mixed phase can be nucleated spontaneously with sufficient speed) one would expect an explosive event in which the star contracts significantly, resulting in the release of a tremendous amount of energy (of order tenths of $M_{\text {sun }}$ ). Cheng and Dai have discussed a similar proposal in which accretion-induced conversion to strange quark matter is suggested as a possible explanation for Gamma-Ray Bursters. 41]

One especially interesting aspect of such a collapse is its potentially turbulent nature. The picture is of a pure K-matter core seeding the mixed phase through several kilometers of material above it. In effect, the K-matter boils off of the outer edge of the second-order core and floats upward to form the mixed phase throughout the entire region of the mixed phase's energetic favorability. This implies an upward and downward transfer of matter that closely resembles turbulent convection, but in which strangeness rather than heat is the substance being convected.

As mentioned at the end of the previous section, more reliable calculations need to be 
performed in order to better understand how the slowness of the weak interactions affect the original nucleation rate estimates based on Langer's formula. We have argued that the correct framework for these future calculations involves the formalism of quantum tunneling (or thermal activation) in a theory with a (nearly) conserved global charge representing strangeness. It is also worth mentioning that the scenario outlined here is the most realistic potential application of the Q-ball nucleation formalism developed in [36],37]; theorists up to now have relied on supersymmetric models to find possible theories containing charged scalars supporting non-topological solitons.

\section{Acknowledgements}

Sanjay Reddy, Eduardo Fraga, and Guy Moore are acknowledged for helpful discussions, though any inaccuracies in the present paper are purely the responsibility of the author. This work is supported in part by a National Science Foundation Graduate Research Fellowship and by the US Department of Energy grant DE-FG03-00ER41132. 


\section{REFERENCES}

[1] J.C. Collins and M.J. Perry Phys. Rev. Lett. 34, (1975) 1353

[2] C. Alcock, E. Farhi, A. Olinto Astrophys. Jl. 310, (1986) 261-72

[3] N.A. Gentile, M.B. Aufderheide, G.J. Mathews, F.D. Swesty, G.M. Fuller Astrophys. Jl. 414, (1993) 701-11

[4] D. Kaplan and A. Nelson Phys. Lett. 175B, (1986) 57

[5] N. K. Glendenning Phys. Rev. D 46, (1992) 1274

[6] J.S. Langer Ann. Phys. (NY) 54, (258-275) 1969

[7] A.D. Linde Nucl. Phys. B216, (1983) 421-445

[8] S. Alamoudi et al Phys. Rev. D 60, (1999) 125003

[9] M.L. Olesen and J. Madsen Phys. Rev. D 49, (1994) 2698-2702

[10] H. Heiselberg hep-ph/9501374

[11] K. Iida and K. Sato Prog. Theor. Phys. 98 (1997) 277-282

[12] P. Shukla, A.K. Mohanty, and S.K. Gupta Phys. Rev. C 62, (2000) 054904

[13] N. K. Glendenning, and J. Schaffner-Bielich, Phys. Rev. Lett. 81, (1998) 4564

N. K. Glendenning, and J. Schaffner-Bielich, Phys. Rev. C 60, (1999) 025803

[14] B. Serot and J.D. Walecka, Advances in Nucl. Physics, 16, edited by J.W. Negele and E. Vogt (Plenum, New York, 1986)

[15] N. K. Glendenning, Compact Stars: Nuclear Physics, Particle Physics and General Relativity , (Springer-Verlag, New York, 1997)

[16] J.A. Pons, S. Reddy, P. Ellis, M. Prakash, and J.M. Lattimer Phys. Rev. C 62, (2000) 034803

[17] R. Knorren, M. Prakash, P.J. Ellis Phys. Rev. C 52, (1995) 3470-82

[18] M. Prakash et al. Phys. Rep. 280, (1997) 1

[19] E. Friedman, A. Gal, and C.J. Batty Nucl. Phys. A579, (1994) 578

A. Cieply, E. Friedman, A. Gal, J. Mares nucl-th/0104087

[20] A. Ramos and E. Oset Nucl. Phys. A671, (2000) 481

[21] M. Christiansen, N.K. Glendenning, and J. Schaffner-Bielich Phys. Rev. C 62, (2000) 025804

[22] J.A. Pons, J.A. Miralles, M. Prakash, and J.M. Lattimer Astrophys. Jl. 553, (2001) 382-393

[23] T. Norsen and S. Reddy Phys. Rev. C 63, (2001) 065804

[24] M.B. Christiansen and N.K. Glendenning Phys. Rev. C 56, (1997) 2858

[25] D.Q. Lamb et al. Nucl. Phys. A411, (1983) 449-473

[26] D.G. Ravenhall, C.J. Pethick, and J.R. Wilson Phys. Rev. Lett. 50, (1983) 2066-9

[27] H. Heiselberg, C.J. Pethick, and E.F. Staubo Phys. Rev. Lett. 70, (1993) 1355-9

[28] J. Lorenzana, C. Castellani, and C. DiCastro cond-mat/0010092

[29] T. Muto, T. Tatsumi, and N. Iwamoto Phys. Rev. D 61, (2000) 063001

T. Muto, T. Tatsumi, and N. Iwamoto Phys. Rev. D 61, (2000) 083002

[30] Z. Dai, T. Lu, and Q. Peng Phys. Lett. 319B, (1993) 199-202

[31] S.K. Ghosh, S.C. Phatak, and P.K. Sahu Nucl. Phys. A596, (1996) 670-83

[32] K. Griest, E.W. Kolb, and A. Massarotti Phys. Rev. D 40, (1989) 3529

[33] D. Metaxas Phys. Rev. D 63, (2001) 083507

[34] S. Coleman Nucl. Phys. B262, (1985) 263

[35] K.M. Benson and L.M. Widrow Nucl. Phys. B353, (1991) 187 
[36] K. Lee Phys. Rev. Lett. 61, (1988) 263

[37] K. Lee Phys. Rev. D 50, (1994) 5333

[38] W. Zhang, T.E. Strohmayer, and J.H. Swank Astrophys. Jl. 482, (1997) L167

[39] M.H. van Kerkwijk astro-ph/0001077

[40] Jerome A. Orosz and Erik Kuulkers astro-ph/9901177

[41] K.S. Cheng and Z.G. Dai Phys. Rev. Lett. 77, (1996) 1210

[42] E.M. Lifshitz and L.P. Pitaevskii Physical Kinetics (Pergamon Press, 1981) 